\title{
DEFICIENT VALUES OF ENTIRE FUNCTIONS AND THEIR DERIVATIVES
}

\author{
DAVID DRASIN, ZHANG GUANGHOU, \\ YANG LO AND ALLEN WEITSMAN ${ }^{1}$
}

\begin{abstract}
Let $f(z)$ be entire and of finite order, $f^{(n)}$ be the $n$th derivative, and $\Delta_{n}(f)=\Sigma \delta\left(a, f^{(n)}\right)$, the sum of all deficient values of $f^{(n)}$. The authors show that $\Delta_{n}(f)$ can be strictly increasing.
\end{abstract}

Let $f(z)$ be entire of order $\rho<\infty$, and for $0<j<\infty$ let

$$
\Delta_{j}(f)=\sum_{|a|<\infty} \delta\left(a, f^{(j)}\right)
$$

where $f^{(0)}=f$ and $f^{(j)}$ is the $j$ th derivative. Using the relation [4, p. 104] $\Sigma \delta(a, f)<$ $\delta\left(0, f^{\prime}\right)$, it is clear that $\Delta_{j}(f)$ is nondecreasing in $j$ while $\Delta_{j}(f)<1$ for all $j$. Professor W. H. J. Fuchs [7, p. 167] recently asked if it is possible that $\Delta_{j}(f)$ be strictly increasing. In this paper we give an affirmative answer. More precisely, we have the stronger

TheOREM. Let $c_{j k}\left(j=0,1,2, \ldots ; k=1,2, \ldots, K_{j} ; 1 \leqslant K_{j} \leqslant \infty\right)$ be finite complex numbers, with $c_{j k} \neq c_{j k^{\prime}}\left(k \neq k^{\prime}\right)$. Given $\frac{1}{2}<\rho<\infty$, and an increasing sequence $\left\{n_{j}\right\}$ of integers, there exists an entire function $f(z)$ of order $\rho$, mean type, such that

$$
\delta\left(c_{j k}, f^{\left(n_{j}\right)}\right)>0
$$

for all $j$ and $k$.

Recently, two of us [8] proved that if $\Delta=\lim \Delta_{j}(f)=1$, then $\Delta_{j}(f) \equiv 1$ for $j>j_{0}(f)$. In the example here $\Delta$ is considerably less than 1 .

Our proof is based on N. Arakeylan's method [2] which produces entire functions of finite order having an infinite set of deficient values. Here, we have a set of deficient functions rather than numbers, but Arakelyan's method is sufficiently flexible to adapt to this situation. The restriction $\rho>\frac{1}{2}$ is essential, since if $\rho<\frac{1}{2}$, then $\Delta_{j}(f) \equiv 0$ for all $j$.

Received by the editors April 28, 1980 and, in revised form, September 4,1980; presented to the Society, January 8, 1981.

1980 Mathematics Subject Classification. Primary 30D35; Secondary 30D30, 30D20, 30E10.

${ }^{1}$ The authors thank Purdue University for this opportunity to collaborate. Drasin and Weitsman acknowledge partial support from the National Science Foundation.

(C) 1981 American Mathematical Society 0002-9939/81/0000-0369/\$02.50 


\section{Preliminary propositions.}

Proposition 1 (Mergelyan [6, p. 125]). Let $\mathcal{L}: z=z(t), 0<t<1$, be a simple rectifiable curve of length $L, z(0)=a, z(1)=b$. If $d>0$ and $0<\varepsilon<1$, there exists a polynomial $P(z)$ such that

$$
\left|\frac{1}{z-a}-P\left(\frac{1}{z-b}\right)\right|<\varepsilon
$$

holds except in a d-neighborhood of $\mathcal{L}$ and also ${ }^{2}$

$$
\left|P\left(\frac{1}{z-b}\right)\right|<\exp \left\{\left(1+\log \left(1+\frac{1}{\varepsilon d}\right)\right) e^{A(L / d)+A}\right\} \quad(|z-b|>d) .
$$

Proposition 2 (Mergelyan [5, p. 61]). Let $f(z)$ be analytic in the sector $|\arg z|<\alpha / 2$, let the number $\rho$ satisfy the condition $0<\rho<\pi / \alpha$, and $\varepsilon>0, \eta>0$ be any numbers. Then there exists an entire function $G(z)$ with

$$
|f(z)-G(z)|<\varepsilon \exp \left(-|z|^{p}\right)
$$

in the sector $|\arg z|<\alpha / 2-\eta$ and

$$
\log |G(z)|<(1+r)^{\pi /(2 \pi-\alpha)}\left\{K+k \max _{0<t<k r+1} \frac{t^{\rho}+\log ^{+} M(t, f)}{(1+t)^{\pi /(2 \pi-\alpha)}}\right\}
$$

in the whole plane; in (1.4) $k$ is a constant depending on $\eta, K$ depends on $\varepsilon$ and $\eta$, and $M(t, f)=\max \left|f\left(t e^{i \theta}\right)\right|(|\theta|<\alpha / 2)$.

\section{Proof of the theorem.}

2.1. It is no loss of generality to assume $n_{j}=j$.

Choose $0<\alpha<\min (\pi / \rho, 2 \pi-\pi / \rho)$ and $\gamma^{j}(j=0,1,2, \ldots)$ such that

$$
0<\gamma^{0}<\gamma^{1}<\cdots<\alpha / 2
$$

and then, for each $j$, choose

$$
\gamma^{j}<\gamma_{j 1}<\gamma_{j 2}<\cdots<\gamma_{j K_{j}}<\gamma^{j+1}
$$

Then we let

$$
\begin{aligned}
\gamma_{j,-k} & =-\gamma_{j k}, \quad\left(j=0,1,2, \ldots ; k=1,2, \ldots, K_{j}\right) \\
\alpha_{j k} & =\min \left\{\frac{1}{2}\left(\gamma_{j k+1}-\gamma_{j k}\right), \frac{1}{2}\left(\gamma_{j k}-\gamma_{j k-1}\right)\right\}=\alpha_{j,-k},
\end{aligned}
$$

where

$$
\gamma_{j k_{j}+1}=\gamma^{j+1}, \quad \gamma_{j 0}=\gamma^{j} \quad(j=0,1,2, \ldots)
$$

Put

$$
\begin{aligned}
& E_{j k n}=\left\{r e^{i \theta}: 2^{n}<r<2^{n+1},\left|\theta-\gamma_{j k}\right|<(1 / 16) \alpha_{j k}\right\}, \\
& E_{j k n}^{1}=\left\{r e^{i \theta}:(15 / 16) 2^{n}<r<(17 / 16) 2^{n+1},\left|\theta-\gamma_{j k}\right|<(1 / 8) \alpha_{j k}\right\}, \\
& E_{j k n}^{2}=\left\{r e^{i \theta}:(7 / 8) 2^{n}<r<(9 / 8) 2^{n+1},\left|\theta-\gamma_{j k}\right|<(1 / 4) \alpha_{j k}\right\} .
\end{aligned}
$$

We will construct an entire function $f(z)$ which satisfies

$$
|f(z)|<\exp \left\{A\left(|z|^{\rho}+1\right)\right\}
$$

\footnotetext{
${ }^{2}$ Here and henceforth $\boldsymbol{A}$ denotes a generic positive absolute constant.
} 
for all $z$ and, for a positive sequence $\varepsilon_{j k}$ to be determined by (2.5),

$$
\left|f(z)-\left(c_{j k} / j !\right) z^{j}\right|<2 \exp \left\{-A \varepsilon_{j k}|z|^{\rho}\right\}
$$

for

$$
\begin{array}{ll}
z \in E_{j k n}^{1} & \left(n>n_{j k}, n \text { even }\right), \\
z \in E_{j,-k, n}^{1} & \left(n>n_{j k}, n \text { odd }\right) .
\end{array}
$$

The $n_{j k}$ are chosen precisely in (2.15) below. Let $H$ be the set of $(j, k, n)$ which appear in (2.3) and denote a typical element $(j, k, n)$ of $H$ by $h$.

We see that $f(z)$ is our required function. In fact, when $z \in E_{h}$, a disk with center at $z$ and radius $10^{-2} \alpha_{j k} 2^{n}$ is contained completely in $E_{j k n}^{1}=E_{h}^{1}$. According to Cauchy's inequality and (2.2),

$$
\begin{aligned}
\left|\left(f(z)-\left(c_{j k} / j !\right) z^{j}\right)^{(j)}\right| & <2 \cdot 10^{2 j j !} \frac{\exp \left\{-A \varepsilon_{j k}\left[|z|-(10)^{-2} \alpha_{j k} 2^{n}\right]^{\rho}\right\}}{\left(\alpha_{j k} 2^{n}\right)^{j}} \\
& <2 \cdot 10^{2 j j !} \frac{\exp \left\{-A \varepsilon_{j k}|z|^{\rho}\right\}}{\left(\alpha_{j k} 2^{n}\right)^{j}} \quad\left(z \in E_{h}\right),
\end{aligned}
$$

i.e.

$$
\frac{1}{\left|f^{(j)}(z)-c_{j k}\right|}>\frac{A}{\left(2 \cdot 10^{2 j} j !\right)} \alpha_{j k}^{j} 2^{n j} \exp \left\{A \varepsilon_{j k}|z|^{\rho}\right\} \quad\left(z \in E_{h}\right)
$$

where $h=(j, k, n) \in H$. On noting from (2.1) that

$$
T\left(r, f^{(j)}\right)=m\left(r, f^{(j)}\right) \leqslant m(r, f)+m\left(r, f^{(j)} / f\right)<A\left(r^{\rho}+1\right),
$$

we obtain by integrating over $(|z|=r) \cap E_{h}$

$$
\delta\left(c_{j k}, f^{(j)}\right)>A \alpha_{j k} \varepsilon_{j k}>0 \quad\left(j=0,1,2, \ldots ; k=1,2, \ldots, K_{j}\right) .
$$

2.2. We now construct a function $Q(\zeta, z)$. When $h=(j, k, n) \in H$, let $C_{h}$ be the arc of the circle $|z|=(9 / 8) 2^{n+1}$ linking $\partial E_{h}^{2}$ to the point $z=-(9 / 8) 2^{n+1}$ which does not meet the positive axis, and let $D_{h}$ be the $\left(2^{n-5} \alpha_{j k}\right)$-neighborhood of $C_{h} \cup \partial E_{h}^{2}$. If $\zeta$ is an arbitrary point of $\partial E_{h}^{2}$, we may connect $\zeta$ to $z=-(9 / 8) 2^{n+1}$ by a curve contained in $C_{h} \cup \partial E_{h}^{2}$. This curve has length less than $A \cdot 2^{n}$, so Proposition 1 produces a rational function $Q(\zeta, z)$ with a unique pole at $-(9 / 8) 2^{n+1}$ such that

$$
|Q(\zeta, z)-1 /(\zeta-z)|<\eta_{h} \quad\left(\zeta \in \partial E_{h}^{2}, z \notin D_{h}\right) .
$$

Thus let

$$
\varepsilon_{j k}=\exp \left(-A / \alpha_{j k}\right)
$$

so that $\varepsilon_{j k}>0$ and

$$
\sum_{j, k} \varepsilon_{j k}=\sum_{j, k} \exp \left(-A / \alpha_{j k}\right)<\sum_{j, k} \alpha_{j k} / A<A .
$$

Then we choose

$$
\eta_{h}=\eta_{j k n}=\alpha_{j k}^{-1} 2^{-(j n+2 n+2 j)} \exp \left\{-4^{\rho+1} \varepsilon_{j k} 2^{n \rho}\right\},
$$


and observe that

$$
\begin{array}{r}
\left\{1+\log \left(1+A / \eta_{h} 2^{n} \alpha_{j k}\right)\right\} \exp \left(A / \alpha_{j k}\right)<A 4^{\rho} \varepsilon_{j k} 2^{n \rho} \exp \left(A / \alpha_{j k}\right)<A 4^{\rho} \varepsilon_{j k}^{1 / 2} \\
\left(n>n_{j k}\right) .
\end{array}
$$

Further, recall the choice of $\alpha$ from the beginning of $\$ 2.1$. Then if $|\arg z|<\alpha / 2$, it is clear that $\left|z-\left(-(9 / 8) 2^{n+1}\right)\right|>A 2^{n+1}$. With these choices of $\varepsilon$ and $\eta_{h}$ in (1.2) and (2.4) we have

$$
\begin{gathered}
\left|Q(\zeta, z)-\frac{1}{\zeta-z}\right|<\alpha_{j k}^{-1} 2^{-(j n+2 n+2 j)} \exp \left\{-4^{\rho+1} \varepsilon_{j k} \cdot 2^{n \rho}\right\} \\
\left(\zeta \in \partial E_{h}^{2}, z \notin D_{h}\right), \\
|Q(\zeta, z)|<\exp \left(A 4^{\rho} \varepsilon_{j k}^{1 / 2} 2^{n \rho}\right) \quad\left(|\arg z|<\alpha / 2, \zeta \in \partial E_{h}^{2}\right) .
\end{gathered}
$$

2.3. The next proposition is essentially in [1], [2, p. 96].

Proposition 3. Let $0<\alpha \leqslant \min (\pi / \rho, 2 \pi-\pi / \rho), \gamma_{j k}$ and $\alpha_{j k}$ be as in $\$ 2.1$, and set

$$
\psi(z)=\exp \left(-\varepsilon_{j k} z^{\rho}\right) \quad\left(\left|\arg z-\gamma_{j k}\right|<\frac{3}{4} \alpha_{j k}\right) .
$$

Then there exists a function $\omega(z)$ holomorphic in $|\arg z|<\alpha / 2$ such that

$$
|\omega(z)|<\exp \left(1+|z|^{p}\right) \quad(|\arg z|<\alpha / 2)
$$

and for $h=(j, k, n) \in H$

$$
A<|\omega(z) / \psi(z)|<A \quad\left(z \in \bigcup_{H} E_{h}^{2}\right) .
$$

Proposition 4. We can choose $n_{j k}$ so that if

$$
g(z)=\left(C_{j} / j !\right) z^{j} \quad\left(z \in E_{h}^{2}, n>n_{j k}\right),
$$

there there exists a function $F(z)$ analytic in $|\arg z|<\alpha / 2$ such that

$$
|F(z)|<\exp A\left(|z|^{\rho}+1\right) \quad(|\arg z|<\alpha / 2)
$$

and

$$
\left|F(z)-\left(C_{j k} / j !\right) z^{j}\right|<\left|\exp \left(-\varepsilon_{j k} z^{\rho}\right)\right| \quad\left(z \in E_{h}^{1}, n>n_{j k}\right) .
$$

Proof. Let $\omega(z)$ be the function obtained in Proposition 3. Then if $h=(j k n) \in$ $H$, and

$$
g_{h}(z)=\frac{1}{2 \pi i} \int_{\partial E_{h}^{2}} \frac{g(\zeta)}{\omega(\zeta)} Q(\zeta, z) d \zeta,
$$

Proposition 3 shows that $g_{h}$ is analytic in $|\arg z|<\alpha / 2$. Using (2.6), (2.8), (2.10) and (2.11), we can obtain

$$
\begin{aligned}
\mid g_{h}(z)-\frac{1}{2 \pi i} & \int_{\partial E_{h}^{2}} \frac{g(\zeta) d \zeta}{\omega(\zeta)(\zeta-z)} \mid \\
& <A \int_{\partial E_{h}^{2}}\left|c_{j k}\right||\zeta|^{j} \alpha_{j k}^{-1} \exp \left\{-4^{\rho+1} \varepsilon_{j k} 2^{n \rho}+\varepsilon_{j k}|\zeta|^{\rho}\right\} \frac{|d \zeta|}{2^{(n j+2 n+2 j)}} \\
& <A\left|c_{j k}\right| \alpha_{j k}^{-1} \exp \left(-\varepsilon_{j k} \cdot 2^{n \rho}\right) \cdot 2^{-n} \quad\left(z \notin D_{h}^{1}\right)
\end{aligned}
$$


Choose $n_{j k}$ so that

$$
\alpha_{j k}^{-1} \exp \left(-\varepsilon_{j k} \cdot 2^{n \rho}\right)<\exp \left\{-\left(\varepsilon_{j k} / 2\right) \cdot 2^{n \rho}\right\} \quad\left(n>n_{j k}\right)
$$

and that

$$
A \sum_{H}\left|c_{j k}\right|^{-n}<1
$$

The integral in the left-hand side of (2.14) is given by

$$
\frac{1}{2 \pi i} \int_{\partial E_{h}^{2}} \frac{g(\zeta) d \zeta}{\omega(\zeta)(\zeta-z)}= \begin{cases}0 & \text { if } z \notin E_{h}^{2} \\ g(z) / \omega(z) & \text { if } z \in E_{h}^{2}\end{cases}
$$

When $z \in E_{h}^{2} \cup D_{h}^{1}$, we have $2^{n-1}<|z|<2^{n+2}$, so that

$$
\begin{aligned}
\left|g_{h}(z)\right| & <A\left|c_{j k}\right| 2^{(n+2) j} \exp \left(A 4^{\rho} \varepsilon_{j k}^{1 / 2} 2^{n \rho}\right) \cdot \exp \left(\varepsilon_{j k} 2^{n \rho}\right) 2^{n} \\
& <\left|c_{j k}\right|^{-n} \exp \left(A 4^{\rho} \varepsilon_{j k}^{1 / 2} 2^{n \rho}\right)
\end{aligned}
$$

by (2.7) and Proposition 3.

Consequently, from (2.14), (2.16), (2.17) and (2.18),

$$
G(z)=\sum_{H} g_{h}(z)
$$

is absolutely convergent for every compact region in the angle $|\arg z|<\alpha / 2$ and $G(z)$ is analytic in this angle. Moreover, from (2.14), (2.17) and (2.18), we have

$$
|G(z)|<\sum_{H}\left|g_{j k n}(z)\right|<\exp \left(A\left(1+|z|^{\rho}\right)\right)
$$

and

$$
\left|G(z)-\frac{c_{j k}}{j !} \frac{z^{j}}{\omega(z)}\right|<1 \quad\left(z \in E_{h}^{1}\right) .
$$

Thus if $F(z)=G(z) \omega(z)$, then $F(z)$ satisfies the conditions of Proposition 4.

2.4. In order to complete the proof of the theorem, we apply Proposition 2 . There exists an entire function $f(z)$ such that

$$
|f(z)-F(z)|<\exp \left(-|z|^{p}\right)
$$

in the angle $|\arg z|<\alpha / 2-\eta$ and

$$
\log |f(z)|<(1+r)^{\pi /(2 \pi-\alpha)}\left\{K+k \max _{0<t<k r+1} \frac{t^{\rho}+\log ^{+} M(t, F)}{(1+t)^{\pi /(2 \pi-\alpha)}}\right\}
$$

in the whole plane.

On noting (2.12) and

$$
\max _{0<t<k r+1} \frac{t^{\rho}}{(1+t)^{\pi /(2 \pi-\alpha)}}<A k^{\rho} \frac{r^{\rho}}{(1+r)^{\pi /(2 \pi-\alpha)}}
$$

we have (2.1). In every $E_{h}^{1}$, we obtain from (2.13) and (2.19)

$$
\begin{aligned}
\left|f(z)-\left(c_{j k} / j !\right) z^{j}\right| & <e^{-r^{\rho}}+\left|F(z)-\left(c_{j k} / j !\right) z^{j}\right| \\
& <e^{-r^{\rho}}+\exp \left(-A \varepsilon_{j k} r^{\rho}\right)<2 \exp \left(-A \varepsilon_{j k} r^{\rho}\right) .
\end{aligned}
$$

Thus (2.2) is satisfied and so $f(z)$ is our desired function. 


\section{REFERENCES}

1. N. U. Arakeljan, Entire functions of finite order with an infinite set of deficient values, Soviet Math. Dokl. 7 (1966), 1303-1306.

2. W. H. J. Fuchs, Théorie de l'approximation des fonctions d'une variable complexe, Université de Montréal, 1968.

3. A. A. Goldberg and I. V. Ostrovskii, The distribution of values of meromorphic functions, "Nauka", Moscow, 1970. (Russian)

4. W. K. Hayman, Meromorphic functions, Clarendon, Oxford, 1964.

5. S. N. Mergelyan, Uniform approximations to functions of a complex variable, Uspehi Mat. Nauk 7 (1952), no. 2 (48), 31-122; English transl., Amer. Math. Soc. Transl. 101 (1954), 1-99.

6. __ On the completeness of systems of analytic functions, Uspehi Mat. Nauk 8 (1953), no. 4 (56), 3-63; English transl., Amer. Math. Soc. Transl. (2) 19 (1962), 109-166.

7. Sanford S. Miller, Complex analysis (Proc. Conf. S.U.N.Y. Brockport, 1978), Dekker, New York, 1978.

8. Yang Lo and Zhang Guanghou, Deficient values and asymptotic values of entire functions, Sci. Sinica, Special Issue (II) (1979), 190-203.

Department of Mathematics, PUrdue University, West LafayetTe, Indina 47907

Institute of Mathematics, Academia Sinica, Bejng, China 